\title{
REVIEW
}

\section{Rasagiline and Rapid Symptomatic Motor Effect in Parkinson's Disease: Review of Literature}

\author{
Michele Pistacchi - Francesco Martinello • \\ Manuela Gioulis $\cdot$ Sandro Zambito Marsala
}

To view enhanced content go to www.neurologytherapy-open.com

Received: September 9, 2013 / Published online: December 17, 2013

(c) The Author(s) 2013. This article is published with open access at Springerlink.com

\section{ABSTRACT}

Rasagiline is a monoamine oxidase type- $\mathrm{B}$ inhibitor used as monotherapy or in addition to levodopa in the treatment of Parkinson's disease. Once daily administration of rasagiline makes it easy to use, and allows good

M. Pistacchi and S. Zambito Marsala contributed equally to the article.

Electronic supplementary material The online version of this article (doi:10.1007/s40120-013-0014-1) contains supplementary material, which is available to authorized users.

M. Pistacchi

Department of Neurology, Santorso Hospital,

Vicenza, Italy

F. Martinello

Rehabilitation Hospital of High Specialization,

Neurology and Neurophysiology, Motta di Livenza

(TV), Italy

M. Gioulis · S. Zambito Marsala ( $₫)$

Department of Neurology, San Martino Hospital, Belluno, Italy

e-mail: sandro.zambito@ulss.belluno.it

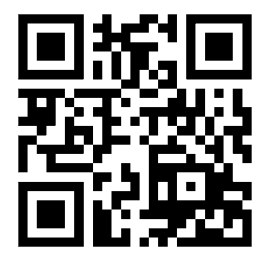

Enhanced content for this article is

available on the journal web site:

www.neurologytherapy-open.com compliance by patients and adherence to therapy. Several multicenter studies have noted the effectiveness of rasagiline on both motor and non-motor symptoms, which require a complex pharmacologic approach, such as cognitive disorders. A recent study also reported a rapid action of rasagiline on motor symptoms. Positive findings have been highlighted by an economic model study. This review analyzes the main studies of rasagiline, with particular attention to the effectiveness of the drug on motor symptoms.

Keywords: Monoamine oxidase type-B inhibitor; Motor symptoms; Neurology; Parkinson's disease; Rasagiline

\section{INTRODUCTION}

Parkinson's disease (PD) is a progressive neurodegenerative disorder characterized by the selective degeneration of dopaminergic neurons in the substantia nigra pars compacta that determines the presence of motor symptoms, namely bradykinesia, muscle rigidity, and resting tremor. However, the 
pathology of the disease often becomes complicated by the presence of non-motor symptoms, which require complex drug treatment, including autonomic disorders, postural instability, bowel and bladder dysfunction, mental disorders (anxiety, apathy, dementia, depression, psychosis), pain, and sleep disorders (daily hypersomnolence, nocturnal akinesia, restless legs syndrome). The majority of drug therapies used in PD aim at increasing the level of striatal dopamine. This can be achieved by administering levodopa, directly stimulating post-synaptic dopamine receptors with dopamine agonists (DA). Another therapeutic strategy involves inhibiting the enzymes responsible for degrading levodopa $[1,2]$.

The enzyme monoamine oxidase (MAO) is a protein of the outer mitochondrial membrane that metabolizes neurotransmitters in the brain and in other tissues. Its inhibition can potentially elevate levels of the major metabolites of neurotransmitters such as dopamine and tyramine [3]. MAO is classified as MAO-A and MAO-B: MAO-A is found mostly in the intestinal tract but also in some presynaptic neurons in the brain, while MAO$\mathrm{B}$ is predominant in the brain, mainly localized in glial cells near the dopaminergic synapses, and regulates both the releasable stores and free levels of free intra-neuronal dopamine [4, 5].

Rasagiline is a selective and irreversible inhibitor of MAO-B, which can be used either as monotherapy or as adjunctive therapy to levodopa or to DA agents in PD [6]. It is five times more potent than selegiline in inhibiting the activity of MAO-B after repeated administration [7]. In healthy volunteers who received a single oral dose of $1,2,5$, or $10 \mathrm{mg}$ rasagiline, maximum inhibition of MAO-B platelet activity, considered to be a marker of brain MAO-B, was observed $1 \mathrm{~h}$ after administration [8]. Significant intergroup differences in MAO-B platelet inhibition favoring rasagiline 2,5 , and $10 \mathrm{mg} /$ day were evident $2 \mathrm{~h}$ after the first dose, while multiple doses of rasagiline $2 \mathrm{mg}$ per day showed $>99 \%$ MAO-B inhibition by day 6 [8]. For all multiple doses of rasagiline, maximum inhibition of MAO-B platelet activity was maintained up to $24 \mathrm{~h}$ after the last administration, and activity returned to baseline levels 2 weeks after the cessation of therapy. In patients with PD, MAOB platelet activity was completely inhibited following 7 days treatment with $0.5,1$, or $2 \mathrm{mg} /$ day rasagiline [9].

This article reviews the main pharmacologic, pharmacodynamic, and pharmacokinetic properties of rasagiline in the treatment of PD.

\section{MATERIALS AND METHODS}

We identified pertinent studies by searching Medline-Pubmed databases (1992-2013) using the key words: rasagiline, MAO-B inhibitors, and Parkinson's disease. We included articles reporting on double-blind, placebo-controlled, randomized studies, open-label randomized studies, controlled studies, and pharmacologic studies. We also considered review articles and research support studies. Articles selected for the review specifically described results of randomized double-blind clinical studies, and long-term clinical trials.

The authors independently selected results focusing on the final outcome measurements, different scales used on clinical or pharmacologic efficacy, statistical significance, and adverse events. Each article was independently reviewed by authors indicating the major interesting data in the field of effectiveness and safety, and disagreements were solved by discussion. 
We also followed the indications of the PRISMA statement: multicenter double-blind, placebo-controlled parallel group, delayed start/ clinical trial, double-blind trial, post hoc analysis, open-label extension, post-marketing observational open-label studies [10].

\section{TEMPO Study}

The TEMPO study was a multicenter, randomized, double-blind placebo-controlled study of rasagiline in 404 patients with earlystage PD [11]. Patients were randomized to receive rasagiline at a dose of 1 or $2 \mathrm{mg}$ /day or placebo. An initial titration period of 1 week was followed by a maintenance period of 25 weeks. The primary measure of efficacy was the change in total Unified Parkinson's Disease Rating Scale (UPDRS) score [12] between baseline and 26 weeks of treatment.

Secondary end points were: cognitive deficits; difficulties with activities of daily living (ADL) [13, 14]; evaluation of motor subscales of the UPDRS, as well as subscores based on symptoms (tremor, rigidity, bradykinesia, instability/gait, and postural changes) [15]. Other secondary variables included changes to the scale of Hoehn and Yahr (H\&Y) [16], the Schwab and England ADL scale [17], the Beck Depression Inventory score [18], the Mini Mental State Examination (MMSE) score [19], timed motor test scores [20], and quality of life (QoL) on the PD Quality of Life (PDQUALIF) scale [21].

Patients who experienced a worsening of scores, $<3$ units in their total UPDRS score from baseline to 26 weeks were classified as responders.

\section{Calculation of the Power of the Study}

According to the Bonferroni method [22], 120 patients per group (360 in total) were required to give a power of between $81 \%$ and $93 \%$ to detect a significant effect of one or both dosages of rasagiline, when treatment with $2 \mathrm{mg} /$ day rasagiline resulted in an improvement of 3 points in UPDRS scores compared with placebo, and treatment with $1 \mathrm{mg} /$ day rasagiline resulted in an improvement in UPDRS scores of between 0 and 3 units.

\section{Results}

Mean [standard deviation (SD)] UPDRS scores at 26 weeks were 24.8 (12.3) in the rasagiline $1 \mathrm{mg} /$ day group, 26.6 (11.8) in the rasagiline $2 \mathrm{mg}$ /day group, and 28.4 (14.3) in the placebo group [11]. The unadjusted changes from baseline were: 0.1 (6.8), 0.7 (5.8), and 3.9 (7.5) in the rasagiline $1 \mathrm{mg} /$ day, $2 \mathrm{mg} /$ day, and placebo groups, respectively. Both active treatment groups showed benefits compared with the placebo group $(P<0.001$ for each comparison).

The analysis of responders who showed a change in UPDRS total score $<3$ units also demonstrated the effects of each active treatment (placebo, 49\%; rasagiline $1 \mathrm{mg} /$ day, $66 \%$, and rasagiline $2 \mathrm{mg} /$ day, $67 \%$, with $P=0.004$ and $P=0.001$ for the 1 and $2 \mathrm{mg}$ rasagiline group vs. placebo, respectively). Of 138 patients in the placebo group, 23 (16.7\%) reached the secondary end point of needing levodopa therapy, compared with 15/134 patients (11.2\%) and 22/132 patients (16.7\%) receiving 1 and $2 \mathrm{mg} /$ day rasagiline, respectively [11].

The Kaplan-Meier analysis showed no statistically significant differences in the start of adjunctive therapy among the three treatment groups. Both groups treated with rasagiline showed significant improvement in PDQUALIF scores compared with placebo. The exploratory analysis suggested that the benefit occurred mainly in the measurement of 
self-image/sexuality subscales, with negligible effects on the social role subscale. Significant benefits were noted in responses to one question comparing present PD symptoms with those experienced 3 months earlier [11].

Adverse events (AEs) were no more frequent in the active treatment groups than in the placebo group. The most commonly observed AEs were infection (16\%) and headache (12\%). Other AEs occurred with a frequency of $<10 \%$. There were no statistically significant differences in the rates of early termination between the treatment groups and the placebo group [11]. Twenty serious AEs (defined as hospitalizations or new malignancies) occurred during the study: four in the placebo group, six in the $1 \mathrm{mg} /$ day rasagiline group, and ten in the $2 \mathrm{mg} /$ day rasagiline group [11]. One patient in the $2 \mathrm{mg}$ /day rasagiline group experienced two serious AEs (hospitalization for depression and delirium).

\section{PRESTO Study}

The PRESTO study was a multicenter, randomized, placebo-controlled double-blind phase III study in 472 patients with PD who experienced at least $2.5 \mathrm{~h}$ of being 'off' (poor motor function) every day despite optimized treatment with levodopa [23].

Patients were randomized to receive rasagiline at a dose of 1 or $0.5 \mathrm{mg} / \mathrm{day}$, or placebo. Eligible patients were aged $>30$ years with idiopathic PD and were in a modified HY stage of $<5$ in the 'off' state. Patients monitored their blood pressure before and after the main meal of the day for 7 days before the baseline, and week 3 , and week 26 visits.

The primary efficacy end point was the change from baseline in mean total daily 'off' time measured through patient diaries, and the average was considered during the treatment period (from week 6, 14, and 26). The secondary end point was the investigator's clinical global impression of improvement, measured on a global scale that included 7 points ranging from 'significantly improved' to 'no change' to 'significantly worsened'., as well as changes from baseline in the UPDRS-ADL scale, and quality of life as measured by the PDQUALIF scale. Additional end points were: changes from baseline in the total average daily 'on' and 'off' times on the Schwab and England ADL scale, and in 'on' times on the UPDRS-ADL scale.

The primary statistical analysis included data from all randomized patients provided with the diary $(n=451)$. To evaluate the effect of patients who withdrew, the analyses were repeated using only patients who completed the study $(n=414)$ and patients who completed all procedures according to the protocol $(n=359)$. The measures of secondary end points were made in the same way as the primary end point. The most common deviations were related to early termination (12\%), less than six acceptable daily diaries (10\%, mainly in patients who left the study prematurely), and change in daily levodopa or other anti-PD dosage by $>20 \%$ from baseline during the last 20 weeks of the study (4\%) [23]. Compliance to treatment was high, as demonstrated by counting pills, with $95 \%$ of patients treated with at least $90 \%$ of the planned doses. Between baseline and week 26, patients treated with placebo decreased their mean \pm SD daily dosage of levodopa from $12 \pm 142 \mathrm{mg}$, while patients treated with $0.5 \mathrm{mg} /$ day rasagiline decreased their dosages by $32 \pm 122 \mathrm{mg}$, and patients treated with $1 \mathrm{mg}$ per rasagiline decreased administration of levodopa by approximately $36 \pm 133 \mathrm{mg}$. The majority of patients were taking other antiparkinsonian drugs, including DA, entacapone, and amantadine [23]. 


\section{Results}

During the treatment period, the average total daily 'off' time decreased from baseline by $1.85 \mathrm{~h}(29 \%)$ in patients treated with $1 \mathrm{mg}$ /day per day rasagiline, $1.41 \mathrm{~h} \mathrm{(23 \% )} \mathrm{with} 0.5 \mathrm{mg} /$ day rasagiline, and $0.91 \mathrm{~h}(15 \%)$ with placebo. Patients treated with $1 \mathrm{mg} /$ day rasagiline had 0.94 h [95\% confidence interval (CI) 0.51-1.36, $P<0.001]$ less 'off' time a day compared with placebo. Patients treated with $0.5 \mathrm{mg} /$ day rasagiline had $0.49 \mathrm{~h}(95 \%$ CI $0.08-0.91$, $P=0.02$ ) less 'off' time compared with placebo. The differences compared to baseline between the groups were maintained throughout the treatment period. There was no difference in treatment effects between the study centers $(P=0.58)$. Compared with placebo, the clinical global impression, UPDRS-ADL score during 'off' time, and UPDRS-motor score during 'on' time improved significantly during treatment with rasagiline [23].

Quality of life, as measured by the PDQUALIF score, showed a trend toward improvement in patients treated with $0.5 \mathrm{mg} /$ day rasagiline $(P=0.07)$, but not with $1 \mathrm{mg} /$ day rasagiline. The social subscale of the PDQUALIF scale showed a benefit for both rasagiline doses compared with placebo, the outlook subscale showed a benefit for the $0.5 \mathrm{mg} /$ day dose, while the function, image, independence, sleep, and urinary subscales showed no difference compared with placebo. Exploratory analyses showed a significant increase in the amount of time 'on' with both doses of rasagiline, corresponding to reductions in the overall time 'off'. In the group treated with $0.5 \mathrm{mg}$ / day rasagiline, there was a greater period of time without troublesome dyskinesia. In the group treated with rasagiline $1 \mathrm{mg} / \mathrm{day}, 32 \%$ of the increase in 'on' time included the presence of troublesome dyskinesias. The rasagiline $1 \mathrm{mg} /$ day group showed a significant improvement in the Schwab and England ADL scale during 'off' times $(P=0.02)$, but a dose of $0.5 \mathrm{mg} /$ day did not produce the same result. The post hoc analysis of UPDRS subscores during the time 'on' showed a significant improvement in rigidity, bradykinesia, and tremor in patients treated with $1 \mathrm{mg} /$ day rasagiline, and in postural instability and in gait and tremor in patients treated with the $0.5 \mathrm{mg} /$ day dose [23].

Adverse events were reported in $87 \%$ of patients treated with placebo, 91\% receiving $0.5 \mathrm{mg}$ /day rasagiline, and 95\% receiving $1 \mathrm{mg} /$ day rasagiline.

These tended to be gastrointestinal AEs and appeared to be dose related. Dyskinesias were reported as an $\mathrm{AE}$ in $10 \%$ of placebo-treated patients, and in $18 \%$ of patients treated with either dose of rasagiline ( $P=0.03$ for combined rasagiline groups vs. placebo). Balance difficulties occurred more often in patients treated with rasagiline, but they did not appear to be dose related. Depression was significantly less common in patients treated with $0.5 \mathrm{mg}$ rasagiline compared with placebo $(P=0.04)$.

There were 22 serious AEs in 14 patients treated with placebo, 42 in 21 patients treated with $0.5 \mathrm{mg}$ rasagiline, and 27 in 18 patients treated with $1 \mathrm{mg}$ rasagiline.

The most common serious AEs were related to accidental injury $(n=6)$, arthritis, worsening PD, melanoma, stroke $(n=3)$, and urinary tract infections $(n=3)$, and none was significantly more common in patients treated with rasagiline than with placebo.

Rasagiline did not have negative effects on blood pressure or heart rate. During treatment, dermatologic examinations revealed three patients with melanoma (1 in the $0.5 \mathrm{mg}$ rasagiline group and 2 patients in the $1 \mathrm{mg}$ rasagiline group). One additional patient was 
identified as having a melanoma before starting the study [23].

\section{LARGO Study}

The LARGO study was a randomized, doubleblind, multicenter 18-week phase III trial of rasagiline in levodopa-treated patients with PD and motor fluctuations [24]. In total, 687 outpatients were randomly assigned to receive oral rasagiline $1 \mathrm{mg} /$ day $(n=231)$, entacapone $200 \mathrm{mg}$ with every levodopa dose $(n=227))$, or placebo $(n=229)$. As a comparator, this trial also included an adjunct entacapone [25-28]. The primary outcome measure was change in total daily 'off' time. The secondary outcome measures included the clinical global improvement (CGI) score [29] and the UPDRS scores. A post hoc analysis of the primary and secondary efficacy variables was undertaken to assess treatment effects stratified by age ( $>70$ or $<70$ years) and according to whether patients received concomitant treatment with DA. Additional exploratory end points included: responder analysis, mean change from baseline in the 'on' state with or without troublesome dyskinesia, and UPDRS-dyskinesia.

\section{Results}

There were no major differences between study groups. Dopamine agonists were the most common class of concomitant drugs for PD (about 60\%, i.e., 130-141 patients in each group). Amantadine and anticholinergic drugs were the other most frequently used therapies with rasagiline. Rasagiline reduced the mean total daily 'off' time from baseline (primary end point) by more than $1 \mathrm{~h}$, almost three times more than the reduction with placebo $(P=0.0001)$ and increased daily 'on' time without troublesome dyskinesia $(0.85 \mathrm{~h}$ vs. placebo $0.03 \mathrm{~h} ; P=0.0005)$. This effect was already evident at the first efficacy assessment (week 6 , adjusted mean change -1.31 vs. -0.27 for placebo; $P=0.0001$ ). Additionally, patients' diaries revealed an accompanying increase in daily 'on' time with the active treatments, most of which was without troublesome dyskinesia. No change in the duration of 'on' time with troublesome dyskinesia was recorded. Responder analysis also supported these findings. This improvement in drug efficacy was accompanied by a small, but significant reduction in levodopa dose with rasagiline (-24 mg/day) compared with a $5 \mathrm{mg} /$ day increase with placebo ( $P=0.0003$ vs. placebo).

At week 18, the clinical global improvement score improved compared with placebo by 0.49 units for rasagiline $(P=0.0001)$. The two UPDRS secondary end points were significantly improved by rasagiline and entacapone: UPDRS-motor ('on' state) and UPDRS-ADL ('off' state). The three UPDRS exploratory subscores measuring dopa-responsive symptoms (tremor, rigidity, and bradykinesia) also significantly improved on rasagiline. UPDRS-dyskinesia scores showed no significant increase when patients were receiving either active treatment compared with placebo.

Three other UPDRS subscores were significantly improved by rasagiline, but not by entacapone: UPDRS-PIGD, UPDRS-freezing (in patients who, at baseline, experienced freezing when walking), and UPDRS-motor in the practically defined 'off' state.

Results of primary efficacy analysis were similar in both active treatment groups in old ( $>70$ years) and young ( $<70$ years) patients. For patients receiving rasagiline, the difference versus that of placebo was $-0.79 \mathrm{~h}$ in the young group, and $-0.76 \mathrm{~h}$ in the old group, indicating similarity between the strata $(P=0.961)$. Significant mean improvements in CGI scores were recorded $(-0.86$ rasagiline; 
$P=0.0001)$. Changes in UPDRS-ADL scores also significantly improved during 'off' time $(-1.71$ vs. placebo; $P=0.0001)$ and motor function during 'on' time $(-2.94$ vs. placebo; $P=0.0001)$.

Rasagiline was well tolerated with a safety profile similar to that of placebo. Importantly, the drug was equally well tolerated in the oldage group ( $\geq 70$ years) with no evidence of increased hallucinations, a common concern with DA. No side effects of dopaminergic treatment were recorded, such as abnormal daytime somnolence, nausea, or leg edema.

Further, rasagiline was given simply, once daily, without titration, both relevant factors for patients who might already be receiving complicated treatment regimens, including the slow titration needed with DA.

\section{Post hoc Analyses of the PRESTO and LARGO Trials}

Post hoc analyses assessed clinical effects of rasagiline $1 \mathrm{mg} /$ day on cardinal $\mathrm{PD}$ symptoms and motor fluctuations in defined patient subgroups using pooled data from PRESTO and LARGO [30]. The effects of rasagiline were measured on individual cardinal PD symptoms during 'on' time and mean change from baseline in daily 'off' time in subgroups of patients who at baseline were receiving only levodopa, were considered 'mild fluctuators' (daily 'off' time $\leq 4 \mathrm{~h}$ ), and who were or were not receiving concomitant DA or catechol-Omethyltransferase inhibitor (COMT-I) therapy.

The consistency between PRESTO and LARGO study designs and outcomes allow data pooling [31]. In both studies, patients could receive stable doses of other dopaminergic medications in addition to levodopa and the study drug. The primary efficacy variable was adjusted mean change from baseline in daily 'off' time, measured by patients in 24-h diaries every $30 \mathrm{~min}$ for 3 days before beginning study treatment and prior to site visits on weeks 6,14 , and 26 in PRESTO and weeks 6, 10, 14, and 18 in LARGO. Patients rated their status as 'on with troublesome dyskinesia, 'on without dyskinesia or without troublesome dyskinesia', 'off,' or 'asleep'. Secondary end points included changes from baseline in the UPDRS scale and ADL subscale score during 'off' time and changes in the UPDRS-motor subscale score during 'on' time. PRESTO and LARGO data were pooled to evaluate the primary and secondary end points in all patients, and in the subgroup of patients who were receiving only levodopa at baseline [32]. Pooled data were also used to evaluate the effect of rasagiline on cardinal symptoms of PD during 'on' time [33] and included analysis of bradykinesia, rigidity, tremor, postural stability, and gait, using the intent-to-treat (ITT) principle.

\section{Results}

When added to already optimized dopaminergic treatment, rasagiline $1 \mathrm{mg}$ /day reduced adjusted mean total daily 'off' time by -0.85 h $(95 \%$ CI -1.16 to $-0.55, P<0.0001)$ compared with placebo. Similarly, compared with placebo, rasagiline $1 \mathrm{mg}$ /day significantly improved mean total daily 'on' time without dyskinesia or with non-troublesome dyskinesia (0.73 h, 95\% CI 0.37-1.08), UPDRS-ADL 'off' score $(-1.56$ points, $95 \% \mathrm{CI}-2.18$ to -0.94$)$, and UPDRS-motor 'on' score (-2.87 points, 95\% CI -3.94 to -1.79$) ; P<0.0001$ for all comparisons. When added to existing dopaminergic treatment, rasagiline $1 \mathrm{mg}$ /day significantly improved all cardinal motor symptoms of $\mathrm{PD}$ compared with placebo during 'on' time $(P<0.05)$, with treatment effects ranging from -0.09 for postural stability to -1.22 for bradykinesia. In addition, 
rasagiline significantly reduced mean 'off' time by $-1.44 \mathrm{~h}(0.20)$, compared with $-0.66 \mathrm{~h}$ (0.19) with placebo (treatment effect $-0.78 \mathrm{~h}$; $P<0.01)$. Similarly, the treatment effect of rasagiline relative to placebo was statistically significant for all secondary end points: -3.74 (0.76) versus -0.17 (0.71), respectively, for baseline UPDRS-motor 'on' scores (treatment effect $-3.57, P<0.001)$, and $-2.25(0.44)$ and -0.59 (0.42), respectively, for baseline mean UPDRS-ADL 'off' scores (treatment effect -1.66 , $P<0.01$ ). The addition of rasagiline to levodopa and potentially other dopaminergic treatments significantly reduced 'off' time by $0.76 \mathrm{~h}$ compared with a $0.22 \mathrm{~h}$ increase with placebo (treatment effect $-0.98 \mathrm{~h} ; P<0.001$ ). Rasagiline $1 \mathrm{mg}$ /day significantly reduced daily 'off' time versus placebo in patients receiving concomitant DA $(-0.92 \mathrm{~h}, P<0.0001)$ and those not $(-0.72 \mathrm{~h}, P<0.0066)$. There was no significant difference between effect sizes of rasagiline-related reductions in daily 'off' time between patients receiving concomitant DA or not $(P=0.5506)$. Similarly, rasagiline $1 \mathrm{mg} /$ day significantly improved all secondary end points in both patients receiving concomitant DA and those not receiving concomitant DA.

\section{Summary}

Post hoc analyses of the PRESTO and LARGO trials showed that rasagiline is a good choice as first adjunct therapy in levodopa-treated patients; rasagiline benefits patients with relatively minor motor symptoms suggestive of early 'wearing off'. The well-established benefits of rasagiline on motor function are related to improvement of all of the cardinal symptoms of $\mathrm{PD}$, and rasagiline added to combination therapy with levodopa and DA or levodopa and COMT-I is well tolerated and further improves symptoms in patients with $\mathrm{PD}$ experiencing motor fluctuations. Therefore, adding rasagiline to an already optimized dopaminergic treatment regimen in patients with relatively mild motor fluctuations $(\leq 4 \mathrm{~h}$ / day at study entry) can reduce mean total daily 'off' time by approximately $25 \%$, whereas total daily 'off' time in placebo-treated patients increased by approximately 7\% during the double-blind studies $(P<0.01$ vs. placebo).

\section{ADAGIO Study}

The ADAGIO study was a double-blind, placebocontrolled, delayed-start study, which randomized 1,176 patients with PD at an early stage and not requiring dopimanergic therapy, to receive rasagiline 1 or $2 \mathrm{mg} /$ day for 72 weeks (early-start group) or placebo for 36 weeks followed by rasagiline 1 or $2 \mathrm{mg} /$ day for another 36 weeks (delayed-start group) [34]. The delayed-start design was used to examine the potential neuroprotective effects of rasagiline in PD. Patients assigned to placebo in phase I who required anti-parkinsonian therapy were included automatically in the active treatment phase (early converters).

The primary analysis of the study included three end points based on the change from baseline in UPDRS scores in the rasagiline versus placebo groups: between the 12th and 32nd week of active treatment; between the 1st and 72 nd week of active treatment; and between the 48th and 72nd week of active treatment. The secondary end point of the study was to compare and estimate the changes to UPDRS score at baseline and at the 72nd week in patients treated with rasagiline $1 \mathrm{mg}$ and $2 \mathrm{mg} /$ day.

\section{Results}

Overall 1,164 patients were included in the first primary end point analysis, and 996 (85\%) were included in the analyses of the second and third 
primary end points. There were no significant differences among the treatment groups at baseline. The mean disease duration from the time of diagnosis was 4.5 months, and the mean total score of 20.4 was obtained on the UPDRS. Patients treated with rasagiline $1 \mathrm{mg}$ /day demonstrated a progression of disease, as measured by UPDRS, lower than in patients treated with placebo from week 12 to 36 $(0.09 \pm 0.02$ points per week vs. $0.14 \pm 0.01$ points per week; $P=0.01)$. In patients receiving rasagiline $1 \mathrm{mg}$ /day, early-start progression was observed at lower average total UPDRS scores between baseline and week 72 compared with the delayed-start group $(2.82 \pm 0.53$ points per week vs. $4.50 \pm 0.56$ points per week; $P=0.02$ ). The estimates of the slope of the UPDRS between week 48 and week 72, at a dose of rasagiline $1 \mathrm{mg} /$ day, showed non-inferiority of the response of the score between the early-start group and the delayed-start group $(0.085 \pm 0.02$ points per week vs. $0.085 \pm 0.02$ points per week; $P<0.001)$. Rasagiline at a dose of $1 \mathrm{mg} /$ day achieved all three primary end points of the study. For patients who received rasagiline $1 \mathrm{mg}$ /day from baseline to the end of phase I, the effectiveness of treatment was superior to placebo $(1.26 \pm 0.36$ points per week of UPDRS vs. $4.27 \pm 0.26$ points per week; $P<0.001)$. For patients who were treated with rasagiline $2 \mathrm{mg} /$ day, a lower progression of disease was observed compared with placebo between week 12 and week 36 on the UPDRS $(0.07 \pm 0.02$ points per week vs. $0.014 \pm 0.01 ; \quad P<0.001)$. Among patients treated with rasagiline $2 \mathrm{mg} /$ day, no significant difference in the overall UPDRS score between baseline and week 72 was noted in patients treated early $(3.47 \pm 0.50$ points per week vs. $3.11 \pm 0.50$ points per week for the delayed-start group; $P=0.60$ ). The estimates of the slope of the UPDRS between week 48 and week 72 , at a dose of $2 \mathrm{mg} /$ day rasagiline, showed non-inferiority of the response between early-start patients and delayed-start patients $(0.094 \pm 0.01$ points per week vs. $0.065 \pm 0.02$ points per week; $P<0.001$ ). The estimated secondary end point for rasagiline $2 \mathrm{mg}$ daily was superior to placebo $(1.11 \pm 0.36$ points per week vs. $4.27 \pm 0.26$ points per week; $P<0.001)$. Rasagiline at a dosage of $2 \mathrm{mg} /$ day did not reach the three primary end points of the study [34].

\section{Post hoc Analysis}

In considering the possibility that the effect of disease modification with rasagiline $2 \mathrm{mg}$ /day may be masked by a mild disease state, the primary and secondary analyses were performed in patients with a UPDRS score $>25.5$ at baseline [34]. Among patients treated with $2 \mathrm{mg}$ /day rasagiline, the difference in the UPDRS scores from baseline to week 72 of the early-start group and the delayed-start group was significantly higher among patients with baseline UPDRS scores in the highest than among patients with scores in the other three quartiles $(P=0.03)$. Therefore, patients in these subgroups can be considered separately. The patients who received 1 or $2 \mathrm{mg} /$ day rasagiline, and who had a baseline score that fell in the highest quartile, met both the primary end point and the secondary end point. The 114 patients who had a UPDRS score in the highest quartile, and who received a dose of $2 \mathrm{mg} /$ day rasagiline in the early-start treatment group, had less progression in overall UPDRS scores between baseline and week $72(-3.63 \pm 1.72$ points; $P=0.04)$. In the 105 patients who had a highest quartile UPDRS score and who received a dose of $1 \mathrm{mg}$ /day rasagiline in earlystart treatment group, had less progression in overall UPDRS scores from baseline to week 72 $(-3.40 \pm 1.66$ points; $P=0.04)$. Patients with UPDRS scores within the lower quartile $(<25.5)$ 
at baseline did not meet the primary end points [34].

\section{Safety}

There were no significant differences in AEs among the study groups, which were: falls, back pain, nasopharyngitis, arthralgia, headache, musculoskeletal pain, nausea or vomiting, hypertension, orthostatic hypotension, somnolence, and hallucination. One patient in the early-start group who received rasagiline at a dose of $1 \mathrm{mg} /$ day had a melanoma at week 72 . No patient had tyramine or serotonin reactions [34].

\section{Summary}

Early treatment with rasagiline at a dose of $1 \mathrm{mg} /$ day provided benefits that were consistent with a possible neuroprotective effect, but early treatment with rasagiline at a dose of $2 \mathrm{mg}$ /day did not. Both doses had beneficial effects on symptoms, as compared to placebo, findings that are similar to those that have been reported previously. Because the two doses were associated with different outcomes, the study results must be interpreted with caution.

\section{Long-Term Outcome of Early Versus Delayed Rasagiline Treatment in Early Parkinson's Disease}

Hauser and colleagues [35] designed a study to evaluate the results of the long-term open-label extension of the TEMPO study. Patients were treated with rasagiline for a maximum of 6.5 years. The objective of the study was to compare progression of long-term illness, assessed by the overall UPDRS score, in patients treated early with rasagiline compared with patients who received it later.

Patients in the TEMPO study $(n=404)$ were randomly assigned to initial treatment with rasagiline (early-start group) or placebo for 6 months followed by rasagiline (delayed-onset group). Those who chose to participate in the open-label extension study $(n=306)$ continued to receive rasagiline as well as other drugs for $\mathrm{PD}$, if necessary. The average duration of the study was $3.6 \pm 2.1$ years; 177 patients took rasagiline for $>5$ years. During the entire 6.5 years of follow-up, the adjusted mean difference in change from baseline in total UPDRS score was 2.5 units [standard error (SE) $1.1 ; P=0.021]$ or $16 \%($ SE $5.7 ; P=0.006)$ for early treatment compared with delayed treatment. The values obtained in the earlystart group were better than in the delayed-start group at all time points. Patients who completed the 52-week double-blind TEMPO study were enrolled into the open-label extension study. Investigators and patients were still blinded to treatment. Patients were enrolled in the TEMPO from November 1997 to June 1999. The results reported here are based on a database reporting the open-label extension until June 2004. Follow-up was 16.5 years estimated from the beginning of the TEMPO study database, but the time of active enrollment in the study for patients ranged according to functional specific start times and possible discontinuation of treatment. During the open-label extension, all patients were initially treated with rasagiline $2 \mathrm{mg} /$ day. Dosage was changed to $1 \mathrm{mg}$ /day in 2000 in line with the TEMPO study.

\section{Results}

Of the 404 patients enrolled in the TEMPO study, 266 were randomly assigned to initial treatment with rasagiline 1 or $2 \mathrm{mg}$, and 138 were randomly assigned to treatment with placebo (delayed-start rasagiline). The treatment groups were comparable with respect to age, sex, and characteristics of PD. A total of 
360 patients who completed the double-blind phase of the TEMPO study were enrolled, and 306 patients chose to participate in the openlabel extension. The average duration of the study for patients in the ITT group was 3.6, 6.0, and 2.1 years for the $1 \mathrm{mg}, 2 \mathrm{mg}$, and placebo groups, respectively, and did not differ between the groups. The average duration of the study was $3.5,6.0$, and 2.2 years, respectively, in the group with early initiation, and 3.6, 6.0, and 2.1 years, respectively in the group with delayed onset; 177 patients continued to receive rasagiline at the time of database lock.

\section{Efficacy}

Changes from baseline to the last visit in the TEMPO study were assessed using the UPDRS score. For the entire period of observation lasting 6.5 years, the adjusted mean difference in change from baseline, expressed as total UPDRS score, was 2.5 units (SE 1.1) $(P=0.021)$ in favor of early-onset group compared to treatment with rasagiline in early delayed. This corresponds to a relative difference in mean percentage change from baseline between groups of $16 \%$ (SE 5.7, $P=0.006$ ). Although the correlation between treatment and time was significant for both analyses $(P=0.0146$ for changing UPDRS score and $P=0.0126$ for the percentage changes of UPDRS score), the value for the early treatment group was numerically better than delayed onset of treatment in all the scores and this correlation reflects the observed variability in the differences between the group that started treatment early and late-start group including a decrease in differences after 1 year compared to 6 months of therapy and an increase in the difference between the groups after 4 years.

The analysis evaluated at intervals of 6 months revealed less significant deterioration, expressed as a percentage of change in total UPDRS score, in the early-start group at $0.5,1.5,2.0,3.0,4.5,5.0$, and 5.5 years $(P<0.05)$. Changes in the UPDRS-ADL motor score were better in the early-start group, with mean differences expressed as a percentage of change from baseline between the groups of 11.9\% (SE 5.9, $P=0.046$ ) and 39.1\% (SE 17.7, $P=0.028)$, respectively.

Of the 177 patients who remained in the study until the opening of the database, 114 were part of the group treated since the very start of the study, and 63 were part of the group with delayed onset, consistent with the ratio of the original randomization. The average duration of treatment with rasagiline was $5.6 \pm 0.4$ years for the group at the beginning of treatment and $5.5 \pm 0.4$ years for the delayed start. The baseline characteristics of the patients who remained in the study until the end of the opening of the database, compared with the ITT population, had longer time from diagnosis to PD and slightly lower than the average total UPDRS score and HY classification. For these 177 patients, the adjusted mean difference in total UPDRS score was 2.42 units (SE \pm 1.04 , $P=0.0218$ ) in favor of the early-start group, corresponding to an average relative difference in change percentage from baseline of $17 \%$ (SE $\pm 5.4, P=0.002)$.

\section{Other Dopaminergic Drugs}

The average length of time from baseline to study time to adding an additional dopaminergic treatment was similar for the early-start group and the delayed-start group (respectively 1.5 years and 1.8 years). Similarly, the percentage of patients who started levodopa or a further additional dopaminergic therapy was comparable for both the early treatment group and for the delayed treatment group. Similarly, the equivalent dosage of levodopa did 
not differ significantly in all 6-monthly intervals between the groups.

\section{Motor Complications}

For the 211 patients for whom data on fluctuations and dyskinesias were collected, the average time period (estimated with the Kaplan-Meier estimates) of the appearance of fluctuations was 6.1 years in the group with early onset $(n=75)$ and 6.0 years in the group with delayed onset $(n=136)$. It was not possible to evaluate the average time period for the appearance of dyskinesias, but the time period for $25 \%$ of the patients being tested was comparable between the two groups: 5.2 compared with 5.9 years. The appearance of the first fluctuations or dyskinesias was 5.5 years in both groups at the beginning and in the early to late start. There were no significant differences using the log-rank test. All patients who reported the appearance of dyskinesias in therapy with rasagiline were previously treated with levodopa and most (75.5\%) were also treated with a DA. Similarly, all fluctuating patients were treated with levodopa (16.9\%), a DA $(15.7 \%)$, or both $(67.4 \%)$.

\section{Long-Term Efficacy of Rasagiline in Early Parkinson's Disease}

Lew et al. [36] conducted a multicenter, doubleblind, placebo-controlled, parallel group delayed-start trial to evaluate the long-term efficacy, safety, and tolerability of treatment with rasagiline monotherapy in patients with PD undergoing treatment for a period of 12 months in the TEMPO trial. Patients were followed for up to 6.5 years with an average of $3.5 \pm 2.1$ years. After the first 12 months, other drugs for PD were added depending on the case. Of the patients who were still in the trial after 2 years, $46 \%$ were kept on monotherapy with rasagiline. Most of the patients received a DA before administration of levodopa as the first additional DA.

In the initial cohort TEMPO study (who had enrolled 398 patients), 360 patients completed the double-blind phase and 306 (85\%) participated in the open-label phase. During the first 12 months, enrolled subjects receiving placebo, or $1 \mathrm{mg}$ or $2 \mathrm{mg}$ per day of rasagiline. During the open-label phase all subjects were administered $2 \mathrm{mg}$ of rasagiline once daily.

The dose of rasagiline was changed to $1 \mathrm{mg} /$ day after 6 months if the efficacy data did not show any difference between the two doses (1 or $2 \mathrm{mg}$ /day). The average duration of treatment with $1 \mathrm{mg}$ /day was $34.1 \pm 17.4$ months (range 5-65 days, 9 months), while the average duration with $2 \mathrm{mg} /$ day was $15.8 \pm 8.3$ months. During the open-label phase, patients were visited approximately every 3 months. UPDRS Parts I to III ('on'), and HY stage were performed. At each visit, the investigator reported the presence of spontaneous AEs and evaluated the possible need for additional therapy.

\section{Results}

The average annual rate of progression assessed by the UPDRS for all patients was equal to 1.93 units (interquartile range 4.99 units). Using the Kaplan-Meier analysis the average time required in the first stage to reach the stage III of H\&Y has not been reached, but the time the 25th percentile required to reach the stage III to the stairs of $H \& Y$ was 5.4 years. The percentage of patients on monotherapy with rasagiline at 2 years was $42 \%$ (95\% CI 36.2-46.9). At 2 years, the remaining $46 \%$ of the cohort had received rasagiline monotherapy.

Baseline UPDRS scores in patients treated with rasagiline monotherapy for 2 years ( $n=121)$ differed significantly compared with patients requiring additional dopaminergic 
therapy $(n=145)$. Patients on monotherapy were older than those needing additional dopaminergic therapy (average age: 62.3 vs. 59.1 years, $P=0.01$ ), had a lower score of disease initiation of treatment with rasagiline (mean UPDRS total 20.0 vs. 28.1 units, $P<0.0001)$, and had a lower UPDRS-motor score (14.1 vs. $19.4, P=0.0001)$, although the same time had elapsed from diagnosis (1.1 vs. 1.3 years, $P>0.05$ ).

At 4 years, $22.5 \%$ of patients were maintained with rasagiline monotherapy, and by 6-6.5 years only $13.0 \%$ remained on monotherapy. At 4 years, approximately onethird of patients were treated with rasagiline plus a DA. The percentage of patients treated with rasagiline plus levodopa was gradually increased (from $10.9 \%$ at 2 years to $17.4 \%$ at 6 years). By 6 years, $60.9 \%$ of patients were on triple therapy with rasagiline, levodopa, and DA.

\section{Efficacy and Tolerability of Rasagiline in Daily Clinical Use: Post-Marketing Observational Study in Patients with Parkinson's Disease}

Reichmann et al. conducted a post-marketing observational study that investigated the efficacy and tolerability of rasagiline in daily clinical practice in Germany [37]. It also examined the subjective assessment of quality of life after treatment with rasagiline. The study included patients with idiopathic PD who received rasagiline (the recommended dose of $1 \mathrm{mg} /$ day) as monotherapy or in combination therapy. The treatment and observation period was approximately 4 months.

\section{Outcome Measures}

Outcome measures, including the change from baseline, were assessed on the Columbia
University Rating Scale (CURS) [38], the UPDRS subscale for fluctuation and daily 'off' time (daily diaries of patients) [12], and the PD Questionnaire-39 (PDQ-39) [39]. Adverse drug reactions (ADRs) and AEs were also examined, as well as global clinical efficacy and safety.

The evaluations were performed at baseline, at 4 weeks and 4 months. Results were recorded from medical charts of data collection.

The score of the CURS was based on the evaluation of 13 items: 3 classic elements (tremor, rigidity, bradykinesia) and 10 nonmotor symptoms or motor non-classical symptoms (facial expression, seborrhea, drooling, impaired speech, finger dexterity, foot tapping, arising from chair, posture, postural stability, gait disturbance). Severity was assessed as: 0 (normal) to 4 (severe). An enhancement in the CURS scale was defined as a reduction in score of at least 1 point.

The UPDRS was calculated from the sequence of four parts UPDRS complications scale, which assesses the predictability, the appearance, and the time spent in the 'off' phase.

QoL was examined using the PD-Q39, which consists of 39 items that can be added together to generate eight subscales, and a total score.

In this analysis, the summed scores could range from 0 (no difficulty) to 100 (maximum difficulty). At the final evaluation at 4 months, investigators were asked to rate on a global level the efficacy and tolerability of rasagiline as: (a) very good, (b) good, (c) modest, or (d) poor. In a post hoc analysis, the overall tolerability assessment was also grouped by age ( $<70$ years; $>70$ years) .

\section{Results}

The authors collected data on 754 patients. In total, 545 patients were treated with rasagiline as an adjunctive therapy and 209 patients 
received rasagiline as monotherapy. All patients on adjunctive therapy and 44 patients on monotherapy (21\%) had received treatment for $\mathrm{PD}$ in the 3 months preceding the study period, most often DA and levodopa/dopadecarboxylase (DDI) preparations.

In total, 29 patients were switched from treatment with rasagiline plus selegiline at the beginning of the study, forming the subgroup of 'switch' patients.

In addition to therapies for PD, medications taken concurrently during the study included beta-blockers (36\% of patients), analgesics (22\%), antidepressants (SSRI excluded 14\%), and diuretics (13\%). The average duration of treatment with rasagiline was $118.2( \pm 33.7)$ days for the total study population, 120.3 $( \pm 25.4)$ days for the monotherapy group, and $117.4( \pm 36.4)$ days for the combination therapy group. During the study period, PD combination therapy remained unchanged in $86.6 \%$ of patients. Only 73 patients $(13.4 \%)$ changed concomitant therapy, including 42 patients with an adjusted dose (mainly of levodopa and DA), 24 patients with drugs then suspended (especially DA), and 18 patients with new prescriptions (mainly levodopa and DA). In the subgroup of switch patients, the pretreatment doses of selegiline ranged from 1.25 to $20.0 \mathrm{mg} /$ day, but were more frequently $5.0 \mathrm{mg} /$ day (12/29 patients) or $10.0 \mathrm{mg} /$ day (8/ 29 patients). The duration of treatment with selegiline and rasagiline consisted of a few days (mostly 1-3 days) in the majority of patients (23/29 patients).

\section{Efficacy}

Patients treated with rasagiline showed a significant improvement in total CURS score over a period of 4 months, both as monotherapy (4.1-point improvement) and in combination (4.6-point improvement) groups.
There was also a significant improvement from baseline in the CURS classical PD motor symptoms (sum of tremor, rigidity, and bradykinesia scores single item) and in the non-classical and classical motor symptoms. All individual sub-items of the CURS were improved (i.e., defined as a reduction in score of at least 1 point) in more than $62 \%$ of patients in the monotherapy group, and up to $48 \%$ of patients receiving combination therapy at the final assessment.

\section{Modifications to the UPDRS}

Fluctuations in the UPDRS clinical end point were examined in 489 patients receiving combination therapy, using the available answers to at least one of four questions. Based on this evaluation, the proportion of patients without 'off' periods of wakefulness during the day increased from $33 \%$ to $50 \%$ during the 4-month period of clinical observation. This confirmed a significant difference in the amount of 'off' time between the start and the end of the study $(P<0.001)$. Furthermore, between the clinical examination at baseline and at the final evaluation, the percentage of patients with predictable periods of 'off' fell from 33\% (95\% CI $28.8-37.2$ ) to $24 \%$ (95\% CI 20.2-27.8), and the percentage of patients with unpredictable 'off' periods fell from 43\% (95\% CI 38.9-47.8) to $31 \%$ (95\% CI 26.8-35.0), with the confidence interval indicating a significant difference in both cases $(P<0.05)$. A significant difference was also detected in the percentage of patients who experienced sudden periods of 'off' (even a few seconds), which also fell from 30\% (95\% CI $26.2-34.3$ ) to $16 \%$ (95\% CI 12.6-19.1) during the study $(P<0.05)$.

\section{Periods of Time in Daily 'Off'}

The time period in daily 'off', as recorded in patient diaries, was examined only in patients 
receiving combination therapy. The evaluation of efficacy was based on data from the diaries of 203 patients who completed all three phases of the study. During the study, considering a median daily period of time equal to 'off' significantly decreased from 120 to $45 \mathrm{~min}$ $(P<0.001)$, with a significant reduction as early as the mid-term (1-4 weeks).

\section{Evaluation of Overall Effectiveness}

At the final evaluation, the overall effectiveness of treatment with rasagiline was assessed by the investigators as good or very good in $133 / 199$ patients on monotherapy (67\%) and in 278/510 patients on combination therapy (55\%). PDQ39 data were supplemented by QoL data reported by patients in a subjective analysis. The overall rating to the PDQ-39, and all eight subscales of the PDQ-39, showed a significant improvement from baseline to final assessment with rasagiline as monotherapy or in combination therapy $(P<0.001)$. Most efficacy was observed in the subscales of the PDQ-39: 'stigma' in the activities of daily life (monotherapy), mobility, and emotional feelings (combination therapy), although the most significant improvements were noted in subscales which value mobility and ADL (monotherapy), and supporting social interaction and communication (combination therapy).

\section{Safety and Tolerability}

AEs/ADRs were reported by 5/209 monotherapy patients (2\%) and $46 / 545$ patients in the combined therapy group (8\%). The ADR/AES most commonly reported were: nausea, dizziness, headache, and vomiting. A total of 100 patients $(13.5 \%)$ were withdrawn from the study prematurely [including 56 patients $(7.6 \%)$ who were withdrawn at the visit of the 4 th week].
The discontinuation rate was higher in the combination therapy group compared with the monotherapy group, both during the study [49 patients $(9.1 \%)$ vs. 7 patients (3.4\%)] and at the final evaluation [87 patients (16.2\%) vs. 13 patients (6.4\%)]. The most common cause of study discontinuation in both groups was lack of efficacy [monotherapy: 7 patients (3.3\%); combination therapy: 31 patients $(5.7 \%)]$.

Two patients on monotherapy (1\%) and 27 patients receiving combination therapy (5\%) withdrew from the study due to lack of tolerability. At the final evaluation, the overall tolerability of treatment with rasagiline was assessed by the investigators as good or very good in 193/199 patients in the monotherapy group (97\%) and in $474 / 527$ patients in the combination therapy group (90\%). The post hoc analysis showed that the tolerability of rasagiline (as monotherapy or in combination therapy) did not seem to be influenced by age and was evaluated as good or very good in $387 / 420$ patients $(92 \%)$ aged $<70$ years, and $276 / 302$ patients $(91 \%)$ aged $\geq 70$ years.

\section{Switch to Rasagiline Group}

The subgroup of patients who switched to rasagiline showed a significant improvement in symptoms between baseline and the final evaluation in both classical and non-classical motor scores (CURS total score, $P<0.001$; classical + nonclassical motor score + partial non-motor score, $P=0.002$ ), and in the percentage of patients free from 'off' periods during the day (36\% vs. $48 \%$, respectively). QoL questionnaires showed that these patients had also benefited from an improvement of 6.5 points in the PDQ-39 total score $(P=0.002)$ and significant improvements in specific individual PDQ-39 scores in motility $(P=0.01)$, ADL $(P<0.01)$, emotional score $(P<0.05)$, and 'stigma' $(P=0.001)$. The tolerability to the 
drug in this subgroup of patients was assessed at the final visit as good or very good in $25 / 26$ patients (96\%).

\section{Summary}

Monotherapy or combination therapy with rasagiline was able to improve the symptoms of $\mathrm{PD}$, reduce the amount of 'off' time, improve QoL, and also demonstrated a favorable tolerability. Furthermore, rasagiline had a simple dosing schedule of one tablet, once per day, without titration. These results are consistent with those obtained in clinical studies (TEMPO, PRESTO, and LARGO).

\section{Time to Onset of Anti-parkinsonian Effect}

Wilson et al. [40] studied 272 PD patients to assess the time to onset and magnitude of the effects of rasagiline, either alone or as an adjunct in an open-label study. Timing evaluations were $1,2,4$, and 12 weeks. Patients were divided into two arms: rasagiline $1 \mathrm{mg}$ /day monotherapy $(n=122)$ or add-on rasagiline $0.5 \mathrm{mg}$ (could be increased to $1 \mathrm{mg}$ at the opinion of the investigator; $n=147$ ) Main outcome measures were the evaluation of the 'bradykinesia score' between baseline and 12 weeks of therapy, the CGI score, the Clinical Global Evaluation-Severity of Illness (CGE-SI) score, Schwab and England ADL score, satisfaction/ease-of-use questionnaire (SAEQ) and investigator for patient, and safety and tolerability. Statistical analyses were conducted according to the ITT principle and analysis of covariance (ANCOVA).

\section{Results}

The magnitude of beneficial effect was similar in monotherapy and adjunct therapy patients. No significant dopaminergic side effects, tyramine reactions, or interactions with antidepressants were observed in the 12 -week trial.

\section{Conclusion}

Rasagiline has a measurable beneficial effect on PD symptoms within 1 week of treatment. Rasagiline has a similar magnitude of benefit in monotherapy and adjunct therapy patients. Adverse interactions between antidepressants and rasagiline were not observed in patients in this trial.

\section{Rapid Efficacy of Rasagiline on Motor Symptoms}

Zambito Marsala et al. [41] studied 102 patients (55 males and 47 females) who started treatment with rasagiline as outpatients: 26 patients were PD therapy naïve and 76 received rasagiline as add-on therapy (i.e., were already receiving levodopa and/or DA). The mean $( \pm \mathrm{SD})$ age was $70.4 \pm 8.7$ years (median 71 years). The mean time from PD diagnosis was 4.7 years. The mean UPDRS III total score at baseline was comparable in the two subgroups (23.2 and 22.1 in therapy-naïve and in add-on therapy patients, respectively; $P=0.636$ ). Treatment with rasagiline was associated with statistically significant decreases from baseline in mean UPDRS III total score in the overall sample and in the two subgroups $(P<0.0001$ at week 1 and week $4)$. The mean $( \pm \mathrm{SD})$ change from baseline in the overall sample was $-6.7 \pm 5.3(95 \% \mathrm{CI}-7.8$ to $-5.7)$ at week 1 , and $-8.9 \pm 6.1$ (95\% CI -10.1 to -7.7$)$ at week 4 . Changes from baseline were $-6.7 \pm 4.4(95 \% \mathrm{CI}-8.4$ to -4.9$)$ at week 1 and $-8.8 \pm 5.9(95 \% \mathrm{CI}-11.2$ to -6.4$)$ at week 4 in therapy-naïve patients, and $-6.8 \pm 5.6(95 \% \mathrm{CI}$ -8.0 to -5.5$)$ at week 1 and $-9.0 \pm 6.1$ (95\% CI -10.4 to -7.6$)$ at week 4 in add-on therapy patients. Significant improvements from 
baseline to both week 1 and week 4 were observed in the overall population for all of the examined UPDRS III items $(P<0.001)$. The mean HY score at baseline was significantly higher in the add-on therapy group than in the therapy-naive subgroup $(2.40$ and 1.90, respectively; $P=0.021$ ). A significant decrease in mean HY score from baseline to week 1 and week 4 ( $P<0.0001$ at any time) was observed in the overall sample, as well as in therapy-naïve and in add-on therapy patients. The extent of the improvement from baseline was more marked in add-on therapy patients than in therapy-naïve patients. The mean $( \pm \mathrm{SD})$ change from baseline in the overall sample was $-0.40 \pm 0.58(95 \% \mathrm{CI}-0.51$ to -0.29$)$ at week 1 and $-0.67 \pm 0.61$ (95\% CI -0.81 to $-0.53)$ at week 4 . Changes from baseline were $-0.23 \pm 0.32(95 \% \mathrm{CI}-0.36 .4$ to -0.10$)$ at week 1 and $-0.46 \pm 0.48$ (95\% CI -0.66 to -0.26 ) at week 4 in therapy-naïve patients, and $-0.46 \pm 0.64(95 \% \mathrm{CI}-0.60$ to -0.31$)$ at week 1 and $-0.74 \pm 0.76$ (95\% CI -0.91 to -0.57$)$ at week 4 in add-on therapy patients.

In the analysis by age, based on median value ( $\leq$ or $>71$ years), the mean UPDRS III total score at baseline was significantly higher in patients aged $>71$ years than in the younger cohort (25.9 and 19.0, respectively; $P=0.001$ ). The mean UPDRS III total score significantly decreased from baseline to both week 1 and week 4 in both subgroups $(P<0.0001)$ : the mean decrease from baseline was slightly higher in older patients than in the younger cohort $(-7.7 \pm 6.2$ vs. $-5.8 \pm 3.2$ at Week, and $-10.0 \pm 7.0$ vs. $-7.9 \pm 4.8$ at week 4$)$. However, the difference between subgroups in percentage change from baseline was not statistically significant at both week 1 (mean difference $-2.6 \%$; 95\% CI -9.4 to $4.1 ; P=0.440)$ and week 4 (mean difference -4.5 ; $95 \% \mathrm{CI}-12.5$ to 3.4 ; $P=0.261)$. As for UPDRS III total score, the mean HY score at baseline was significantly higher in the older than in the younger cohort (2.61 and 1.96, respectively; $P<0.001$ ). Significant decreases from baseline in mean HY score were observed in the two subgroups by median age $(P<0.0001)$, with more marked decreases in older than in younger patients $(-0.52 \pm 0.68$ vs. $-0.28 \pm 0.45$ at week 1 . and $-0.83 \pm 0.79$ vs. $-0.52 \pm 0.60$ at week 4$)$. There were no statistically significant differences between subgroups at week 1 (mean difference $7.1 \% ; 95 \% \mathrm{CI}-1.0$ to $12.5 ; P=0.085)$ or week 4 (mean difference $6.8 \%$; $95 \% \mathrm{CI}-2.1$ to 15.8 ; $P=0.134)$ in percentage changes from baseline.

In the analysis by gender, the mean decrease from baseline in total UPDRS III score was comparable in males $(-6.4 \pm 4.6$ at week 1 and $-9.0 \pm 5.9$ at week 4$)$ and females $(-7.1 \pm 6.1$ at week 1 and $-8.9 \pm 6.3$ at week 4 ), while the mean decrease from baseline in mean HY score was slightly higher in females $(-0.47 \pm 0.75$ at week 1 and $-0.71 \pm 0.79$ at week 4$)$ than in males $(-0.34 \pm 0.38$ at week 1 and $-0.63 \pm 0.64$ at week 4 ). If we evaluate patients who showed a benefit $>20 \%$ on the UPDRS III, we notice that patients showed an average improvement equal to $30.6 \%$ (comparing week 1 with baseline) mean $\quad(\mathrm{SD})=-30.64 \quad(17.120) \quad$ and $\quad 41.7 \%$ (comparing week 1 with baseline) mean $(\mathrm{SD})=-30.64$ (17.120). Considering patients aged $>71$ years $(n=50), 44 \quad(88.0 \%)$ were classed as responders versus 6 (12.0\%) nonresponders. In the group of patients aged $\leq 71$ years $(n=52), 42(80.8 \%)$ were responders versus 10 (19.2\%) non-responders (Chi-square $P$ value $=0.315)$.

\section{Pharmacoeconomic Study of Rasagiline}

Hudry et al. [42] conducted a study to evaluate the cost-benefit of rasagiline and entacapone as adjunctive therapy to levodopa compared with 
levodopa alone in patients with PD and motor fluctuations in Finland. The primary analysis was performed according to the social costs as recommended by International Pharmacoeconomic Finns, taking into account both the direct and the indirect costs. It was also performed in a secondary analysis taking into account only the direct costs [43].

\section{Model Pharmacoeconomics}

The study used a Markov model adapted from a model developed originally by Nuijten [44] and adapted from Palmer [45]. The Markov model was chosen because it allows modeling the long-term evolution of chronic diseases classifying patients into a finite number of states (clinical conditions). This model made it possible to simulate the progression of patients through three different clinical conditions. Two of the three clinical conditions had to meet the criteria in which patients spent a percentage of time during 'off' each day, either $25 \%$ or less off-time/day or greater than $25 \%$ off-time/day. The third condition was that of patients reported as 'dead'. The threshold cutoff of $25 \%$ was obtained from a study that showed that the onset of motor fluctuations determines a parallel increase in costs.

In this study, we adopted the structure of the health states of the model of Palmer and chose a time limit of 2 years. The study duration was reduced because it reflects only a limited period in the natural history of the disease, and factors such as the UPDRS and HY scale, autonomic dysfunction, gait disturbance, cognitive impairment, and concomitant or subsequent disease progression have not been taken into account in the model. The extension of the model beyond 2 years could have resulted in poor validity that does not accurately reflect clinical practice. Moreover, the therapeutic management of PD motor fluctuations is difficult to standardize, because the prevalence of comorbidity increases with the progression of the disease. Also, $15-40 \%$ of patients with PD present with psychiatric complications that require additional medications, resulting in admission to a nursing home and therefore higher costs. The model has been limited to a period of time during which the comparison between different strategies of anti-parkinsonian drugs was realistic. The Markov cycle length was 4 months, which would reflect the normal management of PD in Finland, where patients are examined by a doctor about three times a year. Unlike the Palmer model, this model included all PD patients with motor fluctuations if they had started the cycle 1 , to reflect more accurately the target populations defined for rasagiline treatment. Clinical and economic pharmacoeconomic analyses were conducted using the Data TreeAge software, version 4.0 (Tree Age Software, Williamstown, MA). The LARGO trial was a 4-month, multicenter, double-blind, randomized, placebo- and entacapone-controlled, parallelgroup study designed to compare the efficacy, tolerability, and safety of rasagiline versus placebo in patients with treated PD with motor fluctuations. Its design and results have been described elsewhere.

\section{Basic Analysis}

The results and average costs and their SD were calculated according to a stochastic approach (Monte Carlo simulation with 10,000 iterations) with prior distributions to take account of the variability in each parameter [46]. The distribution of the results was drawn up on the basis of 10,000 iterations. The incremental cost-effectiveness ratio (ICER) was calculated in the absence of dominance (i.e., better efficiency and less cost). 


\section{Sensitivity Analysis}

The multivariate probabilistic approach used in this study took into account the uncertainty related to all variables. The best and worst case scenarios were used to test the hypothesis of effectiveness in the standard care group. In the best of cases, patients in the standard care arm could not improve their daily 'off' time in cycle 1 or any additional cycles. At worst, patients in the standard care arm could improve their daily 'off' time in cycles 1-3, just as for patients in the active treatment arm. This approach was the most prudent for treatment with rasagiline from a methodological point of view, but less likely from a clinical point of view. Another sensitivity analysis was to vary the price of rasagiline through a decrease or an increase in percentage of the price of entacapone to assess the impact of a price difference between the two drugs on cost-effectiveness.

\section{Results}

During the evaluation period of 2 years, rasagiline has dominated standard care from a social point of view. Rasagiline has demonstrated an association with a 0.13 more per quality-adjusted life years (QALYs) and in over $55 \%$ of the time spent in the most with $25 \%$ or less of off-time/day compared to standard treatment. The increase in total effectiveness was not insignificant, with cost savings of $€ 930$ per treated patient, but this did not reach statistical significance. If we consider only the direct costs of rasagiline, there was an increase in direct costs of $€ 2130$ compared with standard care. ICERs were $€ 17,800$ per additional QALY gained and $€ 430$ per additional month spent with $25 \%$ or less offtime per day.

During the evaluation period of 2 years from a social point of view, entacapone obtained a better response compared with standard care.
Treatment with entacapone was associated with a 0.12 additional QALYs and 55\% more time spent with $25 \%$ or less of the period in 'off' compared with standard treatment. In total, the cost saving was estimated at $€ 830$ per patient treated. Considering the prospect of direct costs, the ICER was $€ 18,600$ for additional QALYs acquired and $€ 450$ per additional month spent with $25 \%$ or less of the day 'off'.

Both rasagiline and entacapone proved better than the standard treatment when taking into account both direct and indirect costs. The drug costs associated with rasagiline or entacapone represent a significant proportion of the total (16\%) and direct (30\%) costs.

Indirect costs account for about half of total costs for all treatment strategies considering that PD is a cause of early retirement in more than $60 \%$ of cases. The results in the best cases have shown that the increase of effectiveness of rasagiline compared to the norm has reached 0.18 QALYs (14\% increase) and 7.25 months in the time spent with $25 \%$ or less of 'off'/day (an increase of $56 \%$ ). The total saving is $€ 2,900$ per patient. Incremental direct costs of rasagiline versus standard care were reduced to $€ 1,400$ per patient. Similar results were obtained for the comparison of entacapone and standard care. In the best case, both rasagiline and entacapone were the better strategies than standard treatment from a social point of view. In the worst case, the incremental effectiveness of rasagiline compared with standard treatment was 0.06 QALYs and 2.6 additional months spent with $25 \%$ or less of 'off'-time/day in favor of rasagiline. In this scenario, rasagiline had $€ 3,100$ and $€ 1,550$ additional direct and total costs, respectively, compared with standard treatment. Even on pairing the costs in both clinical conditions (i.e., 1:1 ratio), neither rasagiline nor entacapone had better results 
over the other, as they were more effective but also more expensive compared with standard therapy (additional costs of $€ 2,000$ ). Decreasing the price of rasagiline by $20 \%$ compared to the price of entacapone has resulted in a cost saving from $€ 930$ to $€ 1,500$ per patient over 2 years compared with standard care. The price increase of $10 \%$ compared with entacapone also showed a cost saving of $€ 300$ compared with standard treatment.

\section{Conclusions}

Treatment with rasagiline was convenient and cost saving compared with standard treatment alone and compared with levodopa alone from a social point of view. It was not surprising that rasagiline and entacapone achieved similar performance compared with standard care when considering a similar price for both treatments. Rasagiline is an alternative option for the treatment of advanced PD where unmet needs remain very high.

\section{DISCUSSION}

The main clinical support for the use of rasagiline arises from three large-scale studies, investigating rasagiline as monotherapy in patients with early PD (TEMPO) and as adjunct therapy (to levodopa) in patients with moderate to advanced PD (PRESTO and LARGO).

In the 6-month, placebo-controlled TEMPO study, rasagiline significantly improved PD symptoms (including specific measures of motor function, ADL, tremor, and bradykinesia), and positively affected overall illness severity and patient QoL [11]. The subsequent 6-month active treatment phase of this study indicated that early use of rasagiline may be able to delay symptom progression [35], with the beneficial effect of early treatment maintained for up to 6.5 years in the open-label study extension [36]. Data obtained from the ADAGIO study showed similar results relating to delayed symptom progression [34, 47]. Rasagiline also significantly improved cardinal motor symptoms, in patients with more advanced disease, and reduced daily 'off' time by up to $0.94 \mathrm{~h}$ /day versus placebo in patients experiencing motor fluctuations [23, 24]. In all three pivotal clinical studies, rasagiline was well tolerated and was not associated with any specific safety concerns [11, 23, 24].

Specifically in the TEMPO study, the change in total UPDRS score between baseline and the week 26 visit showed significant differences between active treatment and placebo. In addition, a higher proportion of patients in the active treatment groups responded to therapy, as judged by their change in total UPDRS score compared with the placebo group.

From the analysis of the PRESTO study, it could be demonstrated that between baseline and the visit at 26 weeks, rasagiline treatment was well tolerated and was associated with several therapeutic benefits in PD patients with motor fluctuations, despite optimized levodopa, including decreased 'off' time on home diaries completed by patients, and improvement in CGI performed by blinded examiners. Neurologic function improved during 'off' times (ADL scores based on patient reports) and during 'on' times (overall motor, postural instability and gait, rigidity, bradykinesia, and tremor scores based on patient reports and direct examination). These benefits were measurable at the first efficacy assessment 6 weeks after starting treatment and were sustained throughout the treatment period. Benefits tended to be greater in patients treated with $1 \mathrm{mg} /$ day rasagiline compared with $0.5 \mathrm{mg} /$ day, but differences between the two rasagiline dosages were not 
significant for most end points. Observed decreases in daily 'off' time were associated with nearly equal increases in 'on' time.

The results of LARGO trial showed that $1 \mathrm{mg} /$ day rasagiline without titration reduced 'off' time and improved motor symptom control in patients with $\mathrm{PD}$ who were on optimum treatment of levodopa, DA, and other antiparkinsonian therapies, and the effects were achieved with a good safety profile. Rasagiline reduced daily 'off' time by about $20 \%$ daily. Notably, this beneficial effect was independent of age, adjunct use of DA, and adjustments of levodopa dose. The beneficial effect of rasagiline during 'off' time was accompanied by a corresponding increase in 'on' time without troublesome dyskinesia, whereas the duration of 'on' time with troublesome dyskinesia did not change. The clinical relevance of the primary outcome was supported by the CGI measure.

Rasagiline provided a significant improvement in UPDRS-PIGD and UPDRSfreezing scores. These scores, although not fully validated, relate to symptoms such as postural instability and gait problems, which are regarded as poorly responsive to dopaminergic mechanisms and represent another unmet need of treatment for PD [48]. Rasagiline achieved the two main goals of adjunct therapy for PD, reducing disability and decreasing fluctuations. Rasagiline effectively reduced the time spent in the 'off' state and increased 'on' time without raising the frequency of troublesome dyskinesia.

The results of the ADAGIO study [34] showed that the group of patients treated early with $1 \mathrm{mg} /$ day rasagiline reached the three clinical end points set. Only two of the end points were achieved with the $2 \mathrm{mg} /$ day dose. It can be deduced from the ADAGIO study that early treatment with rasagiline $1 \mathrm{mg} /$ day guarantees benefits that are not are obtained with delayed treatment, despite the use of the same drug. These results are consistent with the possibility that rasagiline may have $1 \mathrm{mg} /$ day slowing effects in PD. In post hoc analyses, patients with baseline UPDRS scores in the highest quartile who received either 1 or $2 \mathrm{mg}$ /day rasagiline met all three primary end points. In the subgroup with UPDRS scores in the highest quartile who received rasagiline at a dose of $2 \mathrm{mg} /$ day, patients in the early-start group had less worsening in UPDRS scores from baseline to week 72 than those in the delayed-start group $(-3.63 \pm 1.72$ points, $P=0.04)$.

In patients with UPDRS scores in the highest quartile at baseline who received rasagiline at a dose of $1 \mathrm{mg} /$ day, those in the early-start group had less worsening in the total UPDRS score from baseline to week 72 than patients in the delayedstart group $(-3.40 \pm 1.66$ points, $P=0.04)$.

Patients enrolled in the TEMPO study were evaluated in a further trial to test the long-term efficacy, safety, and tolerability of rasagiline in all patients who received rasagiline as monotherapy during the trial and subsequent open-label extension [49]. The cohort of patients who were treated with rasagiline monotherapy for 2 years was older, with lower UPDRS scores on study entry, than those requiring adjunctive dopaminergics in the same time frame. The sequence of starting with rasagiline and adding a DA before turning to levodopa was the most commonly chosen approach to long-term therapy. The high proportion of patients maintained on rasagiline monotherapy in the first 2 years of this trial (46\%) suggests that initiating rasagiline therapy in early PD patients offers an efficacious and safe alternative strategy for the use of other dopaminergic treatments for a period of time.

The study by Hauser et al. [35] compared the long-term outcome in patients with early-stage PD in the TEMPO study treated with rasagiline 
earlier in life with those treated late in PD. During the extension phase of the study reported here, patients continued to receive rasagiline, and other PD medications could be added and adjusted as necessary. For the entire follow-up period of 6.5 years, the mean difference in change from baseline in total UPDRS scores between early- and delayed-start patients was 2.5 units, corresponding to a mean relative difference of $16 \%$. Similarly, for patients who continued in the study up to database lock, the adjusted mean difference in change in total UPDRS scores was 2.4 units, corresponding to a mean relative difference of $17 \%$ in favor of the early-start rasagiline group. This suggests that early treatment with rasagiline may offer clinical benefits compared to a delay of treatment for 6 months, and these benefits may be enduring and apparent even as patients are treated with other PD medications.

The post-marketing observational study presented investigated the efficacy and tolerability of rasagiline (as monotherapy or in combination therapy) in daily clinical practice [37]. Either as monotherapy or in combination therapy, rasagiline improved symptoms of PD, reduced the 'off' time, and improved severities of the classical motor symptoms of $\mathrm{PD}$ in $42-62 \%$ of patients, which is a clinically meaningful outcome. The severities of nonclassic motor/non-motor symptoms including speech disorder, finger dexterity, and postural stability were improved in $31-58 \%$ of patients, with favorable tolerability. These results are consistent with the TEMPO, PRESTO and LARGO studies.

Finally, the cost-utility model of rasagiline in advanced PD [42] demonstrated clinical benefits of rasagiline over standard treatment associated with an increased time spent with little or no motor fluctuations and an increase in QALYs. Sensitivity analyses showed a greater clinical benefit with rasagiline than with standard treatment. From a social point of view after 2 years, rasagiline as adjunctive therapy to levodopa showed greater efficiency compared with levodopa alone, without additional costs. From the social point of view, rasagiline demonstrated a reduced utilization of healthcare resources as well as a reduction of indirect costs and, therefore, potential cost savings. The results of this study support the use of rasagiline as an alternative to levodopa in patients with PD and motor fluctuations. Even with a different mechanism of action, rasagiline was an alternative therapeutic agent to entacapone at no additional cost to the community.

\section{Neuroprotective Effects}

Preclinical studies $[49,50]$ have shown that MAO-B inhibitors can protect neurons from oxidative stress, apoptosis, and other forms of injury in multiple experimental models. The possibility that rasagiline might have a neuroprotective effect is supported by laboratory studies showing that the drug and its metabolite 1-(R)-aminoindan have antiapoptotic effects and protect neurons from a variety of toxins in various models [51-54]. Neuroprotection in these models appears to be related to a propargyl ring incorporated within the rasagiline molecule rather than to MAO-B inhibition [54, 55]. In multiple cell culture and animal models, rasagiline has a proven neuroprotective effect [56-58]. Rasagiline reduces neuronal loss in animal models of $N$ methyl-4-phenyl-1,2,3,6-tetrahydropyridine (MPTP) administration [59], oxidative stress [60], hypoxic injury [53], cerebral trauma [61], and amyotrophic lateral sclerosis (ALS) [56]. The MAO-inhibition its propargylamine moiety protects mitochondrial viability and the mitochondrial permeability transition pore by 
activating Bcl-2 and protein kinase $\mathrm{C}$, and downregulating pro-apoptotic FAS and Bax $[54,55]$. Rasagiline also increases nerve growth factor, glial cell-derived neurotrophic factor, and brain-derived neurotrophic factor [62].

The most likely mechanism of action of rasagiline is through inhibition of MAO-B leading to slower catabolism of endogenous dopamine. However, other mechanisms are also possible. In addition to the effect of MAO$\mathrm{B}$ on dopamine catabolism, rasagiline possesses an aminoindan metabolite with antiparkinsonian properties [63]. Rasagiline has been shown to protect neurons against a range of experimentally induced neuronal injuries [59, 64] in animal models and exert an antiapoptotic effect in cell culture [60]. Another possible mechanism of action of rasagiline is through slowing the rate of loss of dopaminergic neurons. Therefore, the long-term benefit observed with early initiation of rasagiline could be due to a neuroprotective effect.

\section{Cognition Effects}

The primary effect of rasagiline is to improve the efficiency of dopaminergic transmission, specifically dopaminergic function in the prefrontal cortex, which is known to be depleted of dopamine early in cognitive impairment, but not in demented patients with PD [65]. Several studies have shown that the MAO-B inhibitor selegiline provides some beneficial effects on cognition and may confer beneficial effects on certain aspects of attention and executive functions in non-demented PD patients with cognitive impairment [66, 67]. Recent studies have also confirmed the effects of rasagiline on cognitive function in cognitively impaired, but not demented patients with PD, suggesting that rasagiline may confer beneficial effects on certain aspects of attention and executive functions in non-demented PD patients with cognitive impairment [68].

\section{CONCLUSION}

Rasagiline has shown strong evidence of effectiveness on parkinsonian motor symptoms, good tolerability and ease of use in patients, as monotherapy or as adjunctive therapy. These add to the effects on cognition and to the possible neuroprotective activity of rasagiline. The motor effectiveness produced by rasagiline has been demonstrated in many studies, with durable response, with additional studies showing rapid onset of action. Considering also the simplicity of administration, good compliance, and the low side effects, the importance of rasagiline in the treatment of patients with PD at all stages of the disease is well established.

\section{ACKNOWLEDGMENTS}

Dr. Sandro Zambito Marsala is the guarantor for this article and takes responsibility for the integrity of the work as a whole. No funding or sponsorship was received for this study or publication of this article. This work is the result of data collected by all the authors; the final work was written by Dr. Pistacchi and Dr. Sandro Zambito Marsala.

Conflict of interest. Michele Pistacchi, Francesco Martinello, Manuela Gioulis, and Sandro Zambito Marsala declare no conflict of interest.

Compliance with ethical guidelines. The analysis in this article is based on previously conducted studies and does not involve any new studies of human or animal subjects performed by any of the authors. 
Open Access. This article is distributed under the terms of the Creative Commons Attribution Noncommercial License which permits any noncommercial use, distribution, and reproduction in any medium, provided the original author(s) and the source are credited.

\section{REFERENCES}

1. Le Witt PA. Levodopa for the treatment of Parkinson's disease. N Engl J Med. 2008;359:246876.

2. Lees A. Alternatives to levodopa in the initial treatment of early Parkinson's disease. Drugs Aging. 2005;22:731-40.

3. Elmer LW, Bertoni JM. The increasing role of monoamine oxidase type $B$ inhibitors in Parkinson's disease therapy. Expert Opin Pharmacother. 2008;9:2759-72.

4. Hauser RA, Zesiewicz TA. Advances in the pharmacologic management of early Parkinson disease. Neurologist. 2007;13:126-32.

5. Jenner P, Langston JW. Explaining ADAGIO: a critical review of the biological basis for the clinical effects of rasagiline. Mov Disord. 2011;26:2316-23.

6. Oldfield V, Keating GM, Perry CM. Rasagiline: a review of its use in the management of Parkinson's disease. Drugs. 2007;67:1725-47.

7. Youdim MB, Gross A, Finberg JP. Rasagiline $[N-$ propargyl-1 $R(+)$-aminoindan], a selective and potent inhibitor of mitochondrial monoamine oxidase B. Br J Pharmacol. 2001;132:500-6.

8. Thébault JJ, Guillaume M, Levy R. Tolerability, safety, pharmacodynamics, and pharmacokinetics of rasagiline: a potent, selective, and irreversible monoamine oxidase type B inhibitor. Pharmacotherapy. 2004;24:1295-305.

9. Rabey JM, Sagi I, Huberman M, et al. Rasagiline mesylate, a new MAO-B inhibitor for the treatment of Parkinson's disease: a double-blind study as adjunctive therapy to levodopa. Clin Neuropharmacol. 2000;23:324-30.

10. Liberati A, Altman DG, Tetzlaff J, et al. The PRISMA statement for reporting systematic reviews and meta-analyses of studies that evaluate health care interventions: explanation and elaboration. PLoS Med. 2009;6:e1000100.
11. Parkinson Study Group. A controlled trial of rasagiline in early Parkinson disease: the TEMPO study. Arch Neurol. 2002;59:1937-43.

12. Fahn S, Elton RL, Members of the UPDRS Development Committee. Unified Parkinson's disease rating scale. In: Fahn S, Marsden CD, Calne DB, Lieberman A, editors. Recent developments in Parkinson's disease. Florham Park: MacMillan Health Care Information; 1987. p. 153-63.

13. Katz S, Ford AB, Moskowitz RW, Jackson BA, Jaffe MW. Studies of illness in the aged. The index of ADL: a standardized measure of biological and psychosocial function. JAMA. 1963;185:914-9.

14. Lawton MP, Brody EM. Assessment of older people: self-maintaining and instrumental activities of daily living. Gerontologist. 1969;9:179-86.

15. McDermott MP, Jankovic J, Carter J, et al. Factors predictive of the need for levodopa therapy in early, untreated Parkinson's disease. The Parkinson Study Group. Arch Neurol. 1995;52:565-70.

16. Hoehn MM, Yahr MD. Parkinsonism: onset, progression and mortality. Neurology. 1967;17:427-42.

17. Schwab RS, England AC Jr. Projection technique for evaluating surgery in Parkinson's disease. In: Gillingham FJ, Donaldson IML, editors. Third symposium on Parkinson's disease, held at the Royal College of Surgeons of Edinburgh on 20, 21 and 22 May 1968. Edinburgh: E \& S Livingstone; 1969. p. $152-7$.

18. Beck AT, Ward CH, Mendelson M, Mock J, Erbaugh J. An inventory for measuring depression. Arch Gen Psychiatry. 1961;4:561-71.

19. Folstein MF, Folstein SE, McHugh PR. "Mini-Mental State". A practical method for grading the cognitive state of patients for the clinician. J Psychiatr Res. 1975;12:189-98.

20. Langston JW, Widner H, Goetz CG, et al. Core assessment program for intracerebral transplantations (CAPIT). Mov Disord. 1992;7:2-13.

21. Welsh M, McDermott MP, Holloway RG, et al. Development and testing of the Parkinson's disease quality of life scale. Mov Disord. 2003;18:637-45.

22. Hochberg Y. A sharper Bonferroni procedure for multiple tests of significance. Biometrika. 1988;75:800-2.

23. Parkinson Study Group. A randomized placebocontrolled trial of rasagiline in levodopa-treated 
patients with Parkinson disease and motor fluctuations: the PRESTO study. Arch Neurol. $2005 ; 62: 241-8$.

24. Rascol O, Brooks DJ, Melamed E, et al. Rasagiline as an adjunct to levodopa in patients with Parkinson's disease and motor fluctuations (LARGO, Lasting effect in Adjunct therapy with Rasagiline Given Once daily, study): a randomised, double-blind, parallel-group trial. Lancet. 2005;365:947-54.

25. Movement Disorder Society Task Force. Management of Parkinson's disease: an evidencebased review. Mov Disord. 2002;17(Suppl. 4):S1-166.

26. Rascol O, Goetz C, Koller W, Poewe W, Sampaio C. Treatment interventions for Parkinson's disease: an evidence based assessment. Lancet. 2002;359:1589_ 98.

27. Parkinson Study Group. Entacapone improves motor fluctuations in levodopa-treated Parkinson's disease patients. Ann Neurol. 1997;42:747-55.

28. Rinne UK, Larsen JP, Siden A, Worm-Petersen J. Entacapone enhances the response to levodopa in parkinsonian patients with motor fluctuations. Nomecomt Study Group. Neurology. 1998;51:1309-14.

29. National Institute for Mental Health. 028 CGI. Clinical global impressions. In: Guy W, editor. ECDEU assessment manual for psychopharmacology. Rockville: US Department of Health, Education, and Welfare; 1976. p. 217-22.

30. Elmer LW. Rasagiline adjunct therapy in patients with Parkinson's disease: post hoc analyses of the PRESTO and LARGO trials. Parkinsonism Relat Disord. 2013;19:930-6.

31. Schwid SR; for The PRESTO and LARGO Investigators. Once-daily rasagiline produces consistent treatment benefits in US and European studies of Parkinson's disease patients. Eur J Neurol. 2006;13:95 (abstract).

32. Poewe $\mathrm{W}$ for the PRESTO and LARGO Investigators. Rasagiline provides significant benefits as adjunct therapy in patients with moderate Parkinson's disease: subgroup analyses. 10th Congress of the European Federation of Neurological Societies (EFNS) 2006, September 2-5, Glasgow, p. 1193 (Abstract).

33. Elmer LW for the PRESTO and LARGO Investigators. Adjunctive rasagiline provides significant benefits in all cardinal symptoms in patients with moderate to advanced Parkinson's disease. 12th International Congress of Parkinson's Disease and Movement Disorders, 2008, June 22-26, Chicago, p. 666 (Abstract).
34. Olanow CW, Rascol O, Hauser R, et al. A doubleblind, delayed-start trial of rasagiline in Parkinson's disease. N Engl J Med. 2009;361:1268-78.

35. Hauser RA, Lew MF, Hurtig HI, et al. Long-term outcome of early versus delayed rasagiline treatment in early Parkinson's disease. Mov Disord. 2009;24:564-73.

36. Lew MF, Hauser RA, Hurtig HI, et al. Long-term efficacy of rasagiline in early Parkinson's disease. Int J Neurosci. 2010;120:404-8.

37. Reichmann H, Jost WH. Efficacy and tolerability of rasagiline in daily clinical use-a post-marketing observational study in patients with Parkinson's disease. Eur J Neurol. 2010;17:1164-71.

38. Yahr MD, Duvoisin RC, Schear MJ, Barrett RE, Hoehn MM. Treatment of parkinsonism with levodopa. Arch Neurol. 1969;21:343-54.

39. Jenkinson C, Fitzpatrick R, Peto V, Greenhall R, Hyman N. The Parkinson's Disease Questionnaire (PDQ-39): development and validation of a Parkinson's disease summary index score. Age Ageing. 1997;26:353-7.

40. Wilson RE, Seeberger LC, Silver D, et al. Rasagiline: time to onset of antiparkinson effect is similar when used as a monotherapy or adjunct treatment. Neurologist. 2011;17:318-24.

41. Zambito Marsala S, Vitaliani R, Volpe D, et al. Rapid onset of efficacy of rasagiline in early Parkinson's disease. Neurol Sci. 2013;34:2007-13.

42. Hudry J, Rinne JO, Keränen T, Eckert L, Cochran JM. Cost-utility model of rasagiline in the treatment of advanced Parkinson's disease in Finland. Ann Pharmacother. 2006;40:651-7.

43. Finnish Ministry of Social Affairs and Health. Pharmaceutical pricing board. http://www.stm.fi/ en/social_and_health_services/pharmacotherapy. Accessed 4 Dec 2004.

44. Nuijten MJ, van Iperen P, Palmer C, van Hilten BJ, Snyder E. Cost-effectiveness analysis of entacapone in Parkinson's disease: a Markov process analysis. Value Health. 2001;4:316-28.

45. Palmer CS, Nuijten MJ, Schmier JK, Subedi P, Snyder EH. Cost effectiveness of treatment of Parkinson's disease with entacapone in the United States. Pharmacoeconomics. 2002;20:617-28.

46. Briggs AH, Ades AE, Price MJ. Probabilistic sensitivity analysis for decision trees with multiple branches: use of the Dirichlet distribution in a Bayesian framework. Med Decis Making. 2003;23:341-50. 
47. Olanow CW, Rascol O. Early rasagiline treatment slows UPDRS decline in the ADAGIO delayed start study. Ann Neurol. 2008;64(Suppl. 12):S68.

48. Rascol O, Payoux P, Ory F, Ferreira JJ, BrefelCourbon C, Montastruc JL. Limitations of current Parkinson's disease therapy. Ann Neurol. 2003;53(Suppl. 3):S3-15.

49. Gotz ME, Breithaupt W, Sautter J, et al. Chronic TVP-1012 (rasagiline) dose-activity response of monoamine oxidases $\mathrm{A}$ and $\mathrm{B}$ in the brain of the common marmoset. J Neural Transm Suppl. 1998;52:271-8.

50. Finberg JP, Takeshima T, Johnston JM, Commissiong JW. Increased survival of dopaminergic neurons by rasagiline, a monoamine oxidase B inhibitor. NeuroReport. 1998;9:703-7.

51. Bar-Am O, Amit T, Youdim MB. Aminoindan and hydroxyaminoindan, metabolites of rasagiline and ladostigil, respectively, exert neuroprotective properties in vitro. J Neurochem. 2007;103:500-8.

52. Stefanova N, Poewe W, Wenning GK. Rasagiline is neuroprotective in a transgenic model of multiple system atrophy. Exp Neurol. 2008;210:421-7.

53. Mandel S, Weinreb O, Amit T, Youdim MB. Mechanism of neuroprotective action of the antiParkinson drug rasagiline and its derivatives. Brain Res Brain Res Rev. 2005;48:379-87.

54. Weinreb O, Amit T, Bar-Am O, Chillag-Talmor O, Youdim MB. Novel neuroprotective mechanism of action of rasagiline is associated with its propargyl moiety: interaction of Bcl-2 family members with PKC pathway. Ann N Y Acad Sci. $2005 ; 1053: 348-55$.

55. Youdim MB, Wadia A, Tatton W, Weinstock M. The anti-Parkinson drug rasagiline and its cholinesterase inhibitor derivatives exert neuroprotection unrelated to MAO inhibition in cell culture and in vivo. Ann N Y Acad Sci. 2001;939:450-8.

56. Waibel S, Reuter A, Malessa S, Blaugrund E, Ludolph AC. Rasagiline alone and in combination with riluzole prolongs survival in an ALS mouse model. J Neurol. 2004;251:1080-4.

57. Youdim MB, Bar Am O, Yogev-Falach M, et al. Rasagiline: neurodegeneration, neuroprotection, and mitochondrial permeability transition. J Neurosci Res. 2005;79:172-9.

58. Maruyama W, Akao Y, Carrillo MC, Kitani K, Youdium MB, Naoi M. Neuroprotection by propargylamines in Parkinson's disease: suppression of apoptosis and induction of prosurvival genes. Neurotoxicol Teratol. 2002;24:675-82.

59. Heikkila RE, Duvoisin RC, Finberg JP, Youdim MB. Prevention of MPTP-induced neurotoxicity by AGN-1133 and AGN-1135, selective inhibitors of monoamine oxidase-B. Eur J Pharmacol. 1985;116:313-7.

60. Maruyama W, Yamamoto T, Kitani K, Carrillo MC, Youdim M, Naoi M. Mechanism underlying antiapoptotic activity of a (-)deprenyl-related propargylamine, rasagiline. Mech Ageing Dev. 2000;116:181-91.

61. Huang W, Chen Y, Shohami E, Weinstock M. Neuroprotective effect of rasagiline, a selective monoamine oxidase- $\mathrm{B}$ inhibitor, against closed head injury in the mouse. Eur J Pharmacol. 1999;366:127-35.

62. Youdim MB, Maruyama W, Naoi M. Neuropharmacological, neuroprotective and amyloid precursor processing properties of selective MAO-B inhibitor antiparkinsonian drug, rasagiline. Drugs Today (Barc). 2005;41:369-91.

63. Finberg JP, Wang J, Bankiewikz K, Harvey-White J, Kopin IJ, Goldstein DS. Increased striatal dopamine production from L-DOPA following selective inhibition of monoamine oxidase B by $R(+)-N$ propargyl-1-aminoindan (rasagiline) in the monkey. Neural Transm Suppl. 1998;52:279-85.

64. Abu-Raya S, Blaugrund E, Trembovler V, Shilderman-Bloch E, Shohami E, Lazarovici P. Rasagiline, a monoamine oxidase- $\mathrm{B}$ inhibitor, protects NGF-differentiated PC12 cells against oxygen-glucose deprivation. J Neurosci Res. 1999;58:456-63.

65. Elmer L, Schwid S, Eberly S, et al. Rasagilineassociated motor improvement in PD occurs without worsening of cognitive and behavioral symptoms. J Neurol Sci. 2006;248:78-83.

66. Bortolato M, Chen K, Shih JC. Monoamine oxidase inactivation: from pathophysiology to therapeutics. Adv Drug Deliv Rev. 2008;60:152733.

67. Lees AJ. Selegiline hydrochloride and cognition. Acta Neurol Scand Suppl. 1991;136:91-4.

68. Hanagasi HA, Gurvit H, Unsalan P, et al. The effects of rasagiline on cognitive deficits in Parkinson's disease patients without dementia: a randomized, double-blind, placebo-controlled, multicenter study. Mov Disord. 2011;26:1851-8. 